Article

\title{
Antioxidant and Antiglycation Effects of Polyphenol Compounds Extracted from Hazelnut Skin on Advanced Glycation End-Products (AGEs) Formation
}

\author{
Ludovica Spagnuolo (D), Susanna Della Posta, Chiara Fanali, Laura Dugo * $\mathbb{D}$ and Laura De Gara
}

check for updates

Citation: Spagnuolo, L.; Della Posta, S.; Fanali, C.; Dugo, L.; De Gara, L. Antioxidant and Antiglycation Effects of Polyphenol Compounds Extracted from Hazelnut Skin on Advanced Glycation End-Products (AGEs) Formation. Antioxidants 2021, 10, 424. https://doi.org/10.3390/antiox 10030424

Academic Editor:

Alejandro Gugliucci

Received: 8 February 2021

Accepted: 4 March 2021

Published: 10 March 2021

Publisher's Note: MDPI stays neutral with regard to jurisdictional claims in published maps and institutional affiliations.

Copyright: (c) 2021 by the authors. Licensee MDPI, Basel, Switzerland. This article is an open access article distributed under the terms and conditions of the Creative Commons Attribution (CC BY) license (https:// creativecommons.org/licenses/by/ $4.0 /)$.
Unit of Food Science and Nutrition, Department of Science and Technology for Humans and the Environment, Campus Bio-Medico University of Rome, via Álvaro del Portillo 21, 00128 Rome, Italy; 1.spagnuolo@unicampus.it (L.S.); s.dellaposta@unicampus.it (S.D.P.); c.fanali@unicampus.it (C.F.); 1.degara@unicampus.it (L.D.G.)

* Correspondence: 1.dugo@unicampus.it; Tel.: +39-06-22541-9470

\begin{abstract}
The advanced glycation end-products (AGEs) arise from non-enzymatic reactions of sugar with protein side chains, some of which are oxido-reductive in nature. Enhanced production of AGEs plays an important role in the pathogenesis of diabetic complications as well as in natural aging, renal failure, oxidative stress, and chronic inflammation. The aim of this work is to study antiglycation effects of polyphenol compounds extracted by hazelnut skin that represents an example of polyphenols-rich food industry by-product, on AGEs formation. AGEs derived from incubation of bovine serum albumin (BSA) and methylglyoxal (MGO) were characterized by fluorescence. The phenolics identification and total polyphenol content in hazelnut skin extracts were analyzed by HPLC-MS and the Folin-Ciocalteu method, respectively. Antioxidant efficacy was evaluated by monitoring total antioxidant activity to assess the ABTS radical scavenging activity of samples by TEAC assay and oxygen radical absorbance capacity (ORAC) assay, expressed as millimoles of Trolox equivalents per gram of sample. Data here presented suggest that phenolic compounds in hazelnut skin have an inhibitory effect on the BSA-AGEs model in vitro, and this effect is concentrationdependent. The putative role of the hazelnut skin antioxidative properties for hindering AGEs formation is also discussed. Because of AGEs contribution to the pathogenesis of several chronic diseases, foods enriched, or supplements containing natural bioactive molecules able to inhibit their production could be an interesting new strategy for supporting therapeutic approaches with a positive effect on human health.
\end{abstract}

Keywords: advanced glycation end products (AGEs); antiglycation; antioxidant; food chain waste/byproducts hazelnut skin; polyphenols

\section{Introduction}

Nowadays, the positive correlation between consumption of plant food rich in bioactive components and good health is well known. During the last decade, much research focused on the role of plant food in health maintenance and prevention of chronic disease [1,2]. Moreover, food waste products derived from food processing are still quite rich in interesting bioactive compounds, which could be extracted and used to produce nutraceutical supplements and cosmetic products [3]. Hazelnuts represent an interesting source of by-products, producing a big amount of waste material such as leafy covers, skins, and shells. Hazelnuts are typically consumed whole or used as an ingredient in many processed foods. Recently, the study of their composition has gained attention with the aim to add economic value to waste from hazelnut processing [4-7].

A wide investigation on hazelnut by-products showed their antioxidant activity by different tests, revealing as they could potentially be considered an excellent source of natural antioxidants [4]. The main bioactive molecules in hazelnut by-products are phenolic 
compounds, a group of chemical substances widely distributed in plant-derived foods. They include phenolic acids, stilbenes, lignans, and flavonoids [5,8]. Phenolic compounds have been reported to have different beneficial effects on human health, such as antioxidant properties, and, as demonstrated in previous studies, also an anti-glycation function [9]. A recently published review reports the inhibitory effects of polyphenols and plant extracts on the formation of advanced glycation end-products (AGEs) [10]. AGEs are heterogeneous compounds that derive from the reaction of reducing sugars with the free amino groups in proteins, nucleic acids, and lipids in a non-enzymatic Maillard-reaction [11]. The first product of glycation is the instable Schiff base adduct which rearranges to form the Amadori product that breaks down to generate some di-carbonyl compounds such as glyoxal (GO), methylglyoxal (MGO), and deoxyglucosones (3-DG) following both oxidative and nonoxidative pathways. In the final step, irreversible colored compounds called AGEs are formed [12]. Di-carbonyl compounds are very reactive products, and they represent a critical step in AGEs generation because they can also generate hydroxyl aldehydes and the corresponding oxidized acid analogues [13]. For example, MGO can react with lysine residues generating the adducts, through a mechanism that involves intermediates like aldimine that can oxidize through a metal-catalyzed process giving deaminated allysine (adipic semialdehyde). This latter further oxidizes to yield 2-amino adipic acid, which indeed accumulates in oxidative-based disorders [14].

Several colorimetric and fluorimetric methods are available to determine parameters that are indicators of AGE production, such as the modification rate of lysine and arginine side chains, fructosamine content, aggregation state of the modified proteins, and AGE-specific fluorescence [15]. AGEs act through different mechanisms, such as: crosslinking extracellular and intracellular proteins that imply alteration in the biochemical and physiological properties of proteins [16]; the binding to their cell surface receptor RAGE leads to the activation of intracellular signaling cascades causing the transcription of genes that have a central role in the phatogenesis of several diseases and the increase in the production of reactive oxygen species (ROS) [17].

It is worth noting that the accumulation of glycation adducts during aging is involved in several diseases such as diabetes, renal diseases, and Alzheimer's [18]. The inhibition of AGE formation by synthetic aminoguanidine (AG) has been documented. However, the treatment with aminoguanidine in type 1 diabetics has caused serious complications [19]. The search for natural AGE inhibitors could thus represent a valid alternative approach. Indeed, the use of plant extracts showed to inhibit the AGE development more effectively than aminoguanidine. Therefore, phenolic compounds could represent a natural source of glycation inhibitors [10], being able to reduce the production of early Maillard reaction products [20-22]. In vivo and in vitro studies also revealed positive effects of gallic acid on AGE-induced inflammation [23]. Antiglycative effects of different fruit and seed extracts have also been tested, suggesting that green pepper, peach, and promegranate have the highest capability to inhibit AGE formation, while the extract of hazelnut kernels has a more moderate inhibitory capability [24]. However, it is worth noting that hazelnut skin and kernel have different polyphenol content and molecular biodiversity [25-27]. Recently published data indicated that roasted hazelnut skin is a richer source of total phenolics and has the highest antioxidant activity, followed by natural and roasted hazelnuts [7]. The skin is a richer source of these bio-active molecules, at least in quantitative terms [5]. Moreover, hazelnut skin is an important by-product of the food industry, if we consider that more than 160,000 tons of hazelnuts are produced annually only by the Ferrero Hazelnut Company (https: / / www.hazelnutcompany.ferrero.com, accessed on 1 February 2021; Ferrero International S.A. Findel, Luxemburg).

Worldwide hazelnut production in 2019/2020 was roughly 528,070 tons. Taking into account that roughly $67 \%$ of the total fruit weight is comprised of the shell leads to roughly 353,807 tons of hazelnut shells each year [28-30]. Hazelnut skin represents $2.5 \%$ by weight of the raw material, and it is discarded upon roasting. It has been traditionally used as 
animal bedding, but a lot of research underlined its potentiality as a source of natural antioxidants and dietary fibers [31].

Indeed, hazelnut skin represents an interesting source of bioactive molecules for nutraceutical or food supplements production, in a circular economy approach. Valorization of products defined as "waste" can contribute to both solving the problem of waste management and disposal, with a considerable economic advantage for companies, other than being greatly environmental-friendly and offering a vast range of molecules of natural origin easily available and eco-sustainable.

On these bases, the present study aims to investigate the inhibitory effects on AGEs formation of extracts from hazelnut skin. An in vitro model of AGEs formation induced by MGO was set up and evaluated. Qualitative and quantitative phenolic compounds contents and antioxidant capability were also determined for further studying their relationship with AGE-inhibitory activity.

\section{Materials and Methods}

\subsection{Extraction of Polyphenols from Hazelnut Skins}

Hazelnut skins were pulverized mechanically with a blender, and phenolic compounds were extracted according to the procedure reported by Del Rio et al., with some modifications [5]. Two different extraction procedures were applied: one using a $1 \%(v / v)$ aqueous formic acid solution as an extractive solvent to obtain an aqueous extract and one using methanol/water $(75: 25, v / v)$ as an extractive solvent to obtain a methanolic extract.

An amount of $0.5 \mathrm{~g}$ of hazelnut skins was added to $5 \mathrm{~mL}$ of $1 \%(v / v)$ aqueous formic acid solution in a $15 \mathrm{~mL}$ centrifuge tubes and were extracted for $30 \mathrm{~min}$ in an ultrasound bath (Elmasonic S30H, Elma Schmidbauer $\mathrm{GmbH}$, Singen, Germany) at room temperature, frequency of $37 \mathrm{kHz}$, and heating power of $200 \mathrm{~W}$. The tube was then heated at $70{ }^{\circ} \mathrm{C}$ for $1 \mathrm{~h}$ and centrifuged for $10 \mathrm{~min}$ at $2151 \mathrm{~g}$. The procedure was repeated two times and the extracts were pulled, filtered with a $0.45 \mu \mathrm{m}$ filter and stored at $-20^{\circ} \mathrm{C}$. For the methanolic extract, $0.5 \mathrm{~g}$ of hazelnut skins was added to $5 \mathrm{~mL}$ of methanol/H2O $(75: 25, \mathrm{v} / \mathrm{v})$ solution and extracted for $15 \mathrm{~min}$ in an ultrasound bath (Elmasonic S30H, Elma Schmidbauer GmbH, Singen, Germany) and vortexed for $15 \mathrm{~min}$. This procedure was repeated twice. Then, the solution was centrifuged for $10 \mathrm{~min}$ at $2151 \mathrm{~g}$. The supernatant was filtered with a $0.45 \mu \mathrm{m}$ filter and stored at $-20^{\circ} \mathrm{C}$ until analysis. All chemicals and reagents were purchased from Sigma (Sigma-Aldrich, Milan, Italy).

\subsection{HPLC-DAD/MS Analysis of Phenolic Compounds}

Hazelnut skin extracts were analyzed using a Shimadzu Prominence LC-20A instrument (Shimadzu, Milan, Italy) equipped with two LC-20 AD XR pumps, SIL-10ADvp, CTO-20 AC column oven, and DGU-20 A3 degasser coupled to an SPD-M10Avp PDA detector and a mass spectrometer detector (LCMS-2010, Shimadzu, Tokyo, Japan) equipped with electrospray (ESI) interface. The Shimadzu LC solution Ver. 3.7 software (Shimadzu, Version 3.7) was used to acquire MS data. Separation was performed using a Core Shell column $(150 \times 4.6 \mathrm{~mm}$ I.D., $2.7 \mu \mathrm{m}$ d.p.) (Merck KGaA, Darmstadt, Germany), with the mobile phase pumped at a flow rate of $1 \mathrm{~mL} / \mathrm{min}$.

The mobile phase was (A) $1 \%$ aqueous formic acid and (B) acetonitrile. Both phenolic extracts were separated using the following gradient: $t=0^{\prime} 0 \% \mathrm{~B} ; \mathrm{t}=40^{\prime} 30 \% \mathrm{~B} ; \mathrm{t}=41^{\prime} 100 \% \mathrm{~B}$; $\mathrm{t}=48^{\prime} 100 \% \mathrm{~B} ; \mathrm{t}=49^{\prime} 0 \% \mathrm{~B} ; \mathrm{t}=56^{\prime} 0 \% \mathrm{~B}$. The injection volume was $2 \mu \mathrm{L}$. Data were acquired using a DAD in the range $210-400 \mathrm{~nm}$ and the chromatograms were extracted at $360 \mathrm{~nm}$ for a methanolic extract and at $280 \mathrm{~nm}$ for aqueous extract. MS-chromatograms were acquired in negative ionization mode, using the following parameters: nebulizing gas flow rate (N2): $1.5 \mathrm{~mL}$ min-1; event time: $1 \mathrm{~s}$; mass spectral range: $\mathrm{m} / \mathrm{z} 100-800$; scan speed: $1000 \mathrm{amu} / \mathrm{s}$; detector voltage: $1.5 \mathrm{kV}$; interface temperature: $250^{\circ} \mathrm{C}$; CDL temperature: $300{ }^{\circ} \mathrm{C}$; heat block temperature: $300^{\circ} \mathrm{C}$; interface voltage: $-3.50 \mathrm{kV}$; Q-array voltage: $0.0 \mathrm{~V}$; Q-array RF: $150.0 \mathrm{~V}$. 


\subsection{Determination of Total Phenolic Content}

The total polyphenols content of hazelnut skin extracts was measured by the FolinCiocalteau method. Quantification of total phenolic content in food or biological sample is based on the reaction of phenolic compounds with a colorimetric reagent, which allows measurement in the visible spectrum. The F-C assay relies on the transfer of electrons in alkaline medium from phenolic compounds to phosphomolybdic/phosphotungstic acid complexes to form blue complexes that are determined spectroscopically [32]. The absorbance at $765 \mathrm{~nm}$ was measured by microplate reader (Infinite 200 Pro, Tecan, Männedorf, Switzerland) and measures were performed in triplicate.

The aqueous and methanolic extracts, obtained from the procedure previously described, were combined, and dried by rotary evaporator (Eyela, Tokyo, Japan) at $30{ }^{\circ} \mathrm{C}$ to remove solvent. Then, the dry matter was resuspended in $5 \mathrm{~mL}$ of methanol/ $\mathrm{H}_{2} \mathrm{O}$ $(50: 50 ; v / v)$ solution and subsequently analyzed. According to the procedure reported in the literature [27], an aliquot of $20 \mu \mathrm{L}$ of extract or standard compound was mixed with $100 \mu \mathrm{L}$ of Folin reagent in $1580 \mu \mathrm{L}$ of methanol/ $\mathrm{H}_{2} \mathrm{O}(50: 50 ; v / v)$ solution, followed by incubation for $8 \mathrm{~min}$. Then, $300 \mu \mathrm{L}$ of $\mathrm{Na}_{2} \mathrm{CO}_{3} 0,2 \mathrm{~g} / \mathrm{mL}$ solution was added. The absorbance was measured after incubation at room temperature for $2 \mathrm{~h}$, in the dark using a microplate reader (Infinite 200 Pro, Tecan, Italy). The total phenolic content was determined from a standard curve using gallic acid $(0-2000 \mu \mathrm{g} / \mathrm{mL})$ as a standard and expressed as milligrams of gallic acid equivalents per grams of hazelnut fresh weight (mg GAE/g). All chemicals and reagents were purchased from Sigma (Sigma-Aldrich, Milan, Italy).

\subsection{Determination of Antioxidant Activity}

The antioxidant activity of the $\mathrm{MeOH} / \mathrm{H}_{2} \mathrm{O}$ extract of hazelnut skin was determined by the TEAC and ORAC assays, according to a previously described method with some modification $[33,34]$.

The Trolox equivalent antioxidant capacity (TEAC) assay is based on the ability of the antioxidant present in a sample to scavenge the radical cation 2,2'-azinobis (3-ethylbenzothiazoline-6-sulfonic acid) (ABTS) by spectrophotometric analysis. The radical cation ABTS was produced by reacting $7 \mathrm{mM}$ ABTS with $2.5 \mathrm{mM}$ potassium persulfate in aqueous phosphate buffer $(5 \mathrm{mM}, \mathrm{pH}=7,4)$ solution, stored in the dark at room temperature for $16 \mathrm{~h}$. $\mathrm{ABTS}^{+}$is a blue-green chromogen with a characteristic absorption at $734 \mathrm{~nm}$ with an absorbance of $0.70 \pm 0.04$ [33].

Briefly, $10 \mu \mathrm{L}$ of extract solution, gallic acid, or a standard compound were mixed with $190 \mu \mathrm{L}$ of $\mathrm{ABTS}^{++}$solution diluted in phosphate buffer $(5 \mathrm{mM}, \mathrm{pH}=7.4)$ in a 96-multiwell plate. Antioxidant compounds in the reaction medium capture the free radical with a loss of color and therefore a reduction in absorbance, corresponding quantitatively to the concentration of antioxidants present. The absorbance was monitored after $10 \mathrm{~min}$ using a microplate reader (Infinite 200 Pro, Tecan, Männedorf, Switzerland). A calibration curve was prepared with Trolox as a standard $(0-700 \mu \mathrm{M})$. Results were expressed as mmol Trolox equivalent (TE) per grams of hazelnut fresh weight or acid gallic.

Oxygen radical antioxidant capacity (ORAC) is another method used to estimate the total antioxidant capacity (TAC) of food or natural products based on the inhibition of the peroxylradical-induced oxidation initiated by thermal decomposition of azocompounds such as [2-2'-azobis(2-amidino-propane) dihydrochloride (AAPH)]. Fluorescein (FL) is used as the fluorescent probe.

According to the method described previously [34], $50 \mu \mathrm{L}$ of FL (78 nM) and $50 \mu \mathrm{L}$ of sample, blank or standard were placed in a 96-multiwell-plate, which was heated to $37^{\circ} \mathrm{C}$ for $15 \mathrm{~min}$ and then $25 \mu \mathrm{L}$ of AAPH $(221 \mathrm{mM})$ were added in each well.

The fluorescence was measured immediately, and fluorescence intensity (excitation wavelength $485 \mathrm{~nm}$ and an emission wavelength of $535 \mathrm{~nm}$ ) measurements were then taken every $5 \mathrm{~min}$ to $90 \mathrm{~min}$ using a microplate reader (Infinite 200 Pro, Tecan, Männedorf, Switzerland). A calibration curve was prepared with Trolox as a standard $(0-50 \mu \mathrm{M})$. The ORAC values are expressed as mmol Trolox equivalents (TE) per grams of hazelnut fresh 
weight or acid gallic. All chemicals and reagents were purchased from Sigma (SigmaAldrich, Milan, Italy).

\subsection{In Vitro Glycation Assay with BSA-MGO}

AGE-BSA was prepared by reacting BSA with MGO according to the method described by Starowicz et al. with some modifications [35]. Briefly, BSA $(100 \mathrm{mg} / \mathrm{mL})$ and MGO $(500 \mathrm{mM})$ were dissolved separately in PBS $1 \times(\mathrm{pH} 7,4)$. Then, $2 \mathrm{~mL}$ of each sample were prepared by incubating BSA solution $(4 \mathrm{mg} / \mathrm{mL})$ with MGO solution at different concentration (20-30-50-100 mM) in PBS $1 \times(\mathrm{pH} 7.4)$ containing $0.02 \% \mathrm{NaN}_{3}$ (to prevent microbe development) for $168 \mathrm{~h}$ at $37^{\circ} \mathrm{C}$, in the dark. Subsequently, BSA solution at different concentrations $(2-4-10-25-50 \mathrm{mg} / \mathrm{mL})$ with MGO $(20 \mathrm{mM})$ were prepared in the same way. BSA solution without the addition of MGO was incubated under the same conditions and used as a control (BSA-non glycated).

The hazelnut skin extract, a solution of gallic acid or aminoguanidine (25-50-100-200$400-500 \mu \mathrm{g} / \mathrm{mL}$ ), was incubated in the dark with $4 \mathrm{mg} \mathrm{mL}-1$ BSA and $20 \mathrm{mM} \mathrm{MGO}$ (in phosphate buffer, $\mathrm{pH} 7.4$, with $0.02 \%$ sodium azide). For each experimental condition, $2 \mathrm{~mL}$ of sample have been prepared and then incubated at $37^{\circ} \mathrm{C}$ for $168 \mathrm{~h}$. All chemicals and reagents were purchased from Sigma (Sigma-Aldrich, Milan, Italy)

\subsection{Measurement of AGE Fluorescence}

The fluorescence of BSA-MGO model system (AGEs) was measured after incubation as described in detail previously using a microplate reader (Infinite 200 Pro, Tecan, Männedorf, Switzerland) at excitation/emission wavelengths $365 / 440 \mathrm{~nm}$. Additionally, changes in intrinsic protein fluorescence were detected at excitation/emission wavelengths of $280 / 350 \mathrm{~nm}$ [15]. The inhibitory effect of treatment to gallic acid, aminoguanidine or hazelnut extracts was calculated using the following equation:

[\%] Inhibition $=[1-($ fluorescence intensity of extract/fluorescence intensity of blank $)] \times 100$.

Data are expressed considering IC50 concentrations, defined as the amount of extract or standard compound $(\mu \mathrm{g} / \mathrm{mL})$ required to reduce AGE formation by $50 \%$ and were determined by logarithmic regression analyses $(n=3)$ using GraphPad Prism 6 software.

\subsection{Statistical Analysis}

The graphics and all statistical analysis were performed using GraphPad Prism version 6.0. The data were expressed as mean \pm standard deviation of three independent experiments, with at least three technical replicates in each experiment. $p$-value, ${ }^{*} p<0.05$, ${ }^{* *} p<0.01, * * *<0.001$ were considered statistically significant. The significance of difference was calculated using one-way ANOVA and Tukey as post-test or $t$-test of multiple or two comparison, respectively.

\section{Results}

\subsection{HPLC-PDA/MS Quali-Quantitative Analysis of Phenolic Compounds in Methanolic and Aqueous Extracts of Hazelnut Skin}

The chemical characterization of extracted phenolic compounds was carried out by HPLC-PDA/ESI-MS according to experimental conditions previously described. To identify the phenolic compounds, present in the two extracts, the retention time and UV and MS spectra and data available in literature were considered $[5-7,26,36-39]$. Figure 1 shows HPLC-PDA chromatograms of the aqueous extract $(\lambda=280 \mathrm{~nm})$ and methanolic extract $(\lambda=360 \mathrm{~nm})$. 

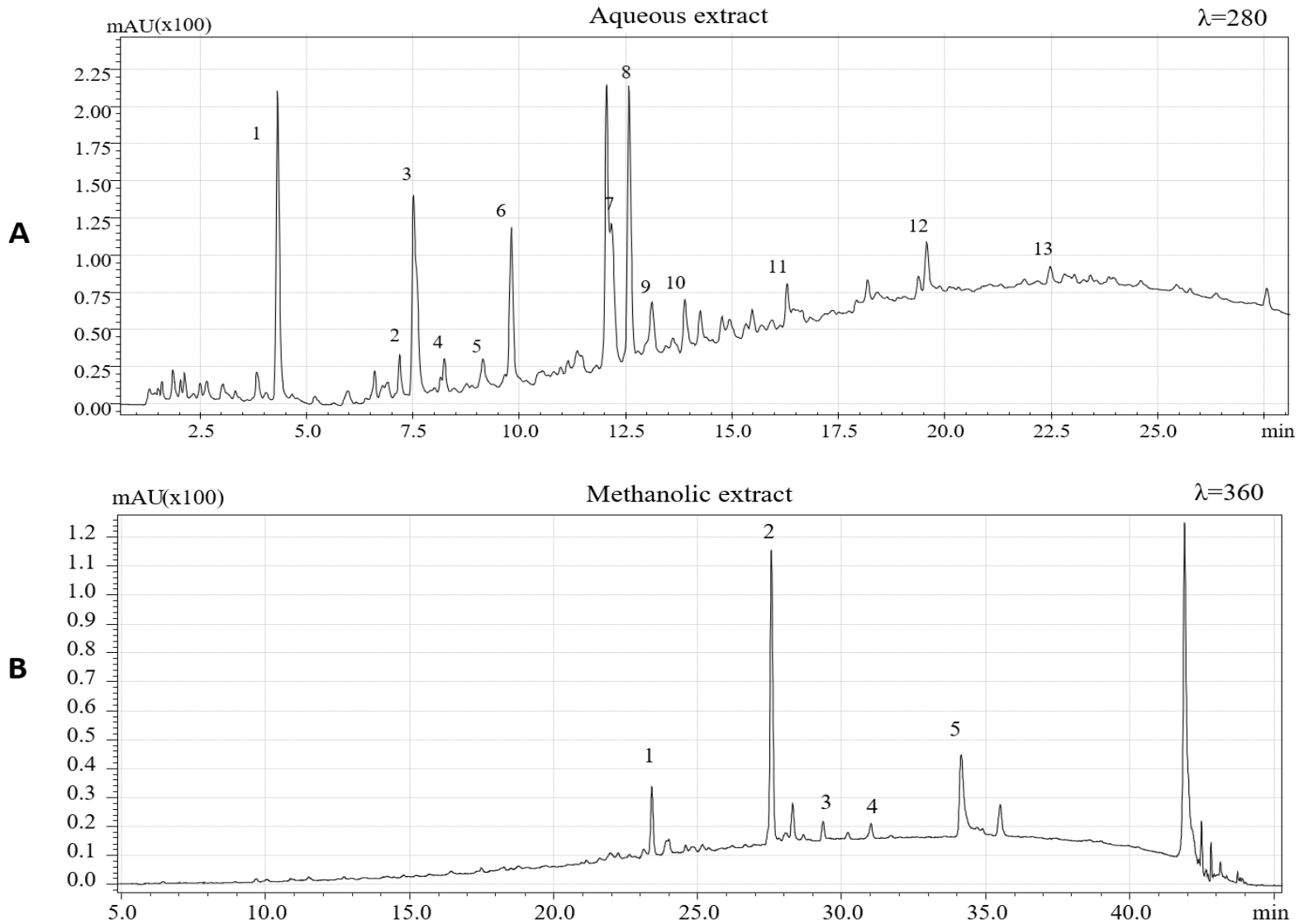

Figure 1. HPLC-PDA chromatograms of hazelnut skin extract: (A) aqueous extract $(\lambda=280)$; (B) methanolic extract $(\lambda=360)$. Peak numbers are referred to Tables 1 and 2.

Table 1. Peaks identification of hazelnut skin aqueous extract through HPLC/ESI-MS.

\begin{tabular}{cccc}
\hline $\mathbf{N}^{\circ}$ & Compound Identified & Retention Time (min) & $\mathbf{~ m / z ~ ( M - H ) ~}$ \\
\hline 1 & Gallic acid & 4.43 & 169 \\
2 & Protocatechuic acid & 7.32 & 153 \\
3 & Procyanidin C2 trimer & 7.55 & 865 \\
4 & Prodelphinidin beta-type dimer & 8.19 & 593 \\
5 & Prodelphinidin beta-type dimer & 9.05 & 593 \\
6 & Prodelphinidin beta-type dimer & 9.59 & 593 \\
7 & Procyanidin beta 1 dimer & 11.96 & 577 \\
8 & (+) Catechin & 12.41 & 289 \\
9 & Procyanidin beta-type trimer & 13.03 & 865 \\
10 & Procyanidin beta-type trimer & 13.83 & 865 \\
11 & (-) epicatechin & 16.20 & 289 \\
12 & Procyanidin Beta-type dimer gallate & 19.55 & 729 \\
\hline
\end{tabular}

Table 2. Peaks identification of hazelnut skin methanolic extract through HPLC/ESI-MS.

\begin{tabular}{cccc}
\hline $\mathbf{N}^{\circ}$ & Compound Identified & Retention Time (min) & $\mathbf{m} / \mathbf{z}(\mathbf{M}-\mathbf{H})^{-}$ \\
\hline 1 & Myricetin rhamnoside & 23.40 & 463 \\
2 & Quercitin 3-0-rhamnoside & 27.52 & 447 \\
3 & Phloretin 2-o-glucoside & 29.30 & 435 \\
4 & Kaempferol rhamnoside & 30.96 & 431 \\
5 & Quercetin & 34.16 & 301 \\
\hline
\end{tabular}

Phenolic compounds identified in aqueous extracts of hazelnut skins are summarized in Table 1. A total of 13 phenolic compounds, being eleven flavan-3-ols and two phenolic 
acids, were identified based on the mass-to-charge ratio $(\mathrm{m} / \mathrm{z})$ of the molecular ion and data available in literature.

Among flavan-3-ol, (+)-catechin, and (-)- epicatechin, having the same mass-tocharge ratio $(\mathrm{m} / \mathrm{z})$, were identified using purified epicatechin as standard molecules and comparing the retention times. These compounds were previously identified in hazelnut skin [5] and are also characteristics of hazelnut shell and kernel as previously reported $[6,26,37]$.

Epicatechin 3-O-gallate, which characterizes the hazelnut shell [6], has been detected with $[\mathrm{M}-\mathrm{H}]^{-}$at $\mathrm{m} / \mathrm{z} 441$, confirming the results obtained by Del Rio et al. [5]. Among procyanidins, one B-type dimer of procyanidins (PCs) was identified, presenting a $[\mathrm{M}-\mathrm{H}]^{-}$ at $\mathrm{m} / \mathrm{z}$ 577. Three procyanidins trimers were detected and one was tentatively identified as procyanidin $\mathrm{C} 2$ based on its elution time preceding procyanidin $\mathrm{B} 1$. One procyanidin gallate trimer with $[\mathrm{M}-\mathrm{H}]^{-}$at $\mathrm{m} / \mathrm{z} 729$ and three isomers of B-type prodelphinidin (PDs) dimers were identified based on their $[\mathrm{M}-\mathrm{H}]^{-}$at $\mathrm{m} / \mathrm{z} 593$. Two isomers of B-type PCs dimers were previously identified in hazelnut shell [6]. Protocatechuic acid $\left([\mathrm{M}-\mathrm{H}]^{-}=153\right)$ and gallic acid $\left([\mathrm{M}-\mathrm{H}]^{-}=169\right)$ were also identified. These two hydroxybenzoic acids were previously identified in hazelnut kernels, hazelnut shell, and hazelnut skins [4-6,37].

In accordance with data reported by Del Rio et al. [5], to extract other less polar flavonoids in hazelnut skin samples, methanolic extraction was performed. Four flavonols and one dihydrochalcone (Table 2) were identified based on their chromatographic and spectrometric behaviors. Three flavonol rhamnosides were identified being myricetin rhamnoside $\left([\mathrm{M}-\mathrm{H}]^{-}=463\right)$, quercetin-3-o-rhamnoside $\left([\mathrm{M}-\mathrm{H}]^{-}=447\right)$ and kaempferol rhamnoside $\left([\mathrm{M}-\mathrm{H}]^{-}=431\right)$. The same compounds were previously detected in hazelnut skin [5] and, except for kaempferol rhamnoside, these compounds have been previously identified also in leaves, shell, and kernels of $C$. avellana L [6,26,37]. One aglycon flavonol, namely, quercetin $\left([\mathrm{M}-\mathrm{H}]^{-}=301\right)$, was also detected. This aglycon have previously been identified in hazelnut skin [5] and in hazelnut shells [6]. Finally, a phenolic compound belonging to the dihydrochalcone subclass, namely, phloretin 2-O-glucoside (phloridzin), was also identified. Specifically, this polyphenol showed a $[\mathrm{M}-\mathrm{H}]^{-}$at $\mathrm{m} / \mathrm{z} 435$. Its presence had already been reported in the hazelnut skin, kernel, and shell $[5,6,26]$.

Figure 2 represents the chemical structure of some phenolic compound, identified in Hazelnut skin extract.
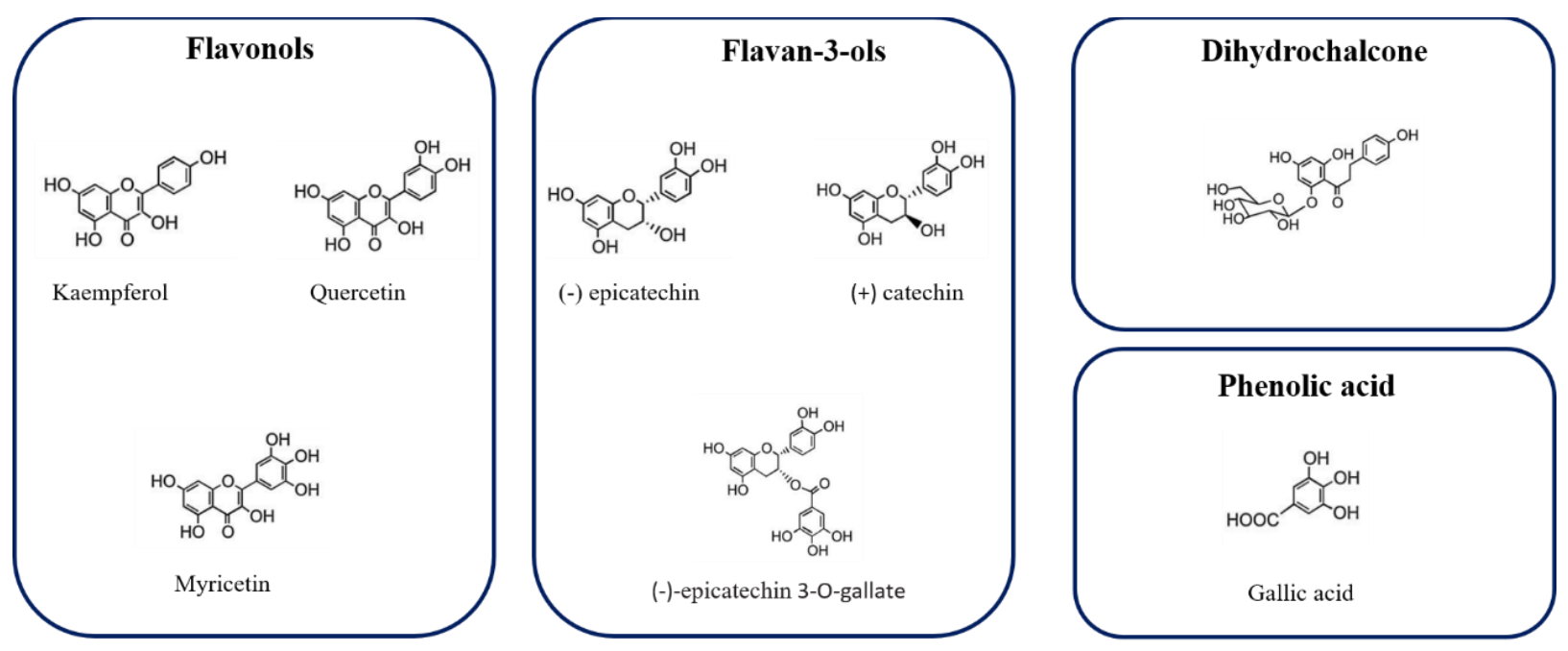

Figure 2. Chemical structure of some phenolic compounds identified in Hazelnut skin extract.

\subsection{Analysis of Phenol Content and Antioxidant Activity in Hazelnut Skin Extract}

Total phenolic content (TPC) and antioxidant activities of hazelnut skin extract were determined by the method previously described. Table 3 shows total phenolic compounds 
and the values of antioxidant power. Our results indicate that polyphenols in the skin represent about $70 \mathrm{mg} \mathrm{GAE} / \mathrm{g}$, (7 g of polyphenol/100 $\mathrm{g}$ of hazelnut skin) in line with literature data [5]. This confirms that hazelnut skin could be a relevant polyphenol source compared to the total phenol content (TPC) in hazelnut kernels that represent about $0.07-0.47 \mathrm{mg}$ GAE/g [26]. According to literature data, a mix of flavan-3-ols like procyanidins with their oligomerization forms, $(+)$-catechin and (-)-epicatechin is the main phenolic component in hazelnut skin (95\%). Flavonols and dihydrochalcones represented an additional 3.5\% while phenolic acids were responsible for less than $1 \%$ of the total identified phenolics in the HPLC-MS/MS method [5].

Table 3. Total phenolic content (TPC) and antioxidant capacity (TEAC and ORAC). TPC of Hazelnut skin expressed as mg of gallic acid per $\mathrm{g}$ of fresh weight. TEAC and ORAC expressed as mmol of trolox per $g$ of fresh weight. All values are expressed as mean $\pm \operatorname{SD}(n=3)$.

\begin{tabular}{cccc}
\hline Compound & TPC & TEAC & ORAC \\
& (mg GAE/g) & (mmol TE/g) & (mmol TE/g) \\
\hline Hazelnut skin $100 \mathrm{mg} / \mathrm{mL}$ & $70.07 \pm 1.38$ & $0.28 \pm 0.03$ & $0.35 \pm 0.02$ \\
Gallic acid $1 \mathrm{mg} / \mathrm{mL}$ & & $10.98 \pm 1.89$ & $42 \pm 3.34$ \\
\hline
\end{tabular}

In relation to the antioxidant capability, it is worth noting that, although the antioxidant capability of skin extract was much lower than that of purified gallic acid (Table 3), no remarkable differences were evident between the two methods of analysis used. On the other hand, the antioxidant capability of gallic acid was about four times higher when measured by the ORAC assay than that observed by TEAC assay. Consequently, the difference in antioxidant capacity between skin extract and purified gallic acid remarkably depend on the used methods. This is probably due to the presence in the skin extract of different molecules able to optimize the scavenging properties against the different oxidant species used in the two methods for the determination of antioxidant capacity. Meanwhile, when a purified molecule is used, different sensitivity/specificity in reacting with other chemical species could explain the remarkable difference in its antioxidant capacity.

In the last few years, accumulating evidence from epidemiological and clinical studies indicates that the daily intake of foods rich in (poly)phenols may possess protective effects in humans [40]. In this context, a positive association between nut, including hazelnut, consumption, and lower risk of all-cause mortality and cardiovascular disease, has been observed [41,42]. Hazelnut skin has been investigated as a functional ingredient rich of bio-active molecules, showing that its addition to coffee, bread, and yogurt improved their physiologically positive effects and antioxidative activity [43-45]. The hazelnut skin extracts also revealed functional activity significantly improving the growth of two probiotic strains (Lactobacillus plantarum P17630 and Lactobacillus crispatus P17631), when added in bacterial media [46]. In vivo, hazelnut skin administration in hamsters improved their plasma lipid profile, following a high fat diet [47]. A recent report also shows a potential role for phenolic hazelnut skin extract as a UV protection booster [48].

\subsection{AGE Quantification}

Several methods are reported in the literature for measuring AGE, many of which use the glycosylation of a standard protein by a standard glycosylation agent $[9,15,49]$. To evaluate whether AGEs formation was dependent on protein (BSA) or intermediate (MGO) concentration, different concentrations of MGO were incubated with BSA at a standard concentration $(4 \mathrm{mg} / \mathrm{mL})$. Figure 3 shows the formation of AGEs during $168 \mathrm{~h}$ of glycation reaction. The presence of total AGEs was characterized by fluorescence with respective excitation and maxima emission at 365 and $440 \mathrm{~nm}$. The BSA-MGO model shows a significant formation of fluorescent AGEs, which is already clear with the lowest MGO concentration uses (20 mM). As expected, in the control sample (non-glycated BSA), a very low fluorescence was observed (Figure 3A). To confirm the MGO-dependent BSA glycation, the alterations in the fluorescence of BSA were analyzed: non-glycated BSA 
showed maximum fluorescence at 280/350 nm, while the 280/350 nm BSA fluorescence intensity strongly decreased as the consequence of MGO treatment (Figure 3B), thus confirming AGEs formation by inducing a conformational change in the protein [15]. AGEs formation with dependence on protein concentration was also confirmed by using a range of 2-50 mg/mL BSA (Figure 4). The comparison between the amount of BSA and BSAMGO complex at $365 / 440 \mathrm{~nm}$ and $280 / 350 \mathrm{~nm}$ indicates that, at $4 \mathrm{mg} / \mathrm{mL}$, most of BSA was converted in the glycated form, while, at higher BSA concentrations, an increased amount of protein remained in the native form. Therefore, in the following set of experiments, $4 \mathrm{mg} / \mathrm{mL}$ BSA was used.

A

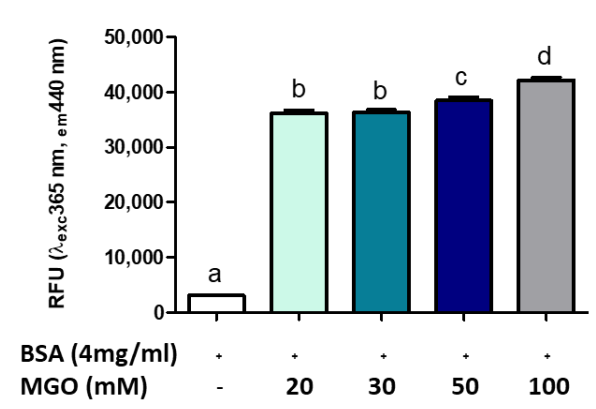

B

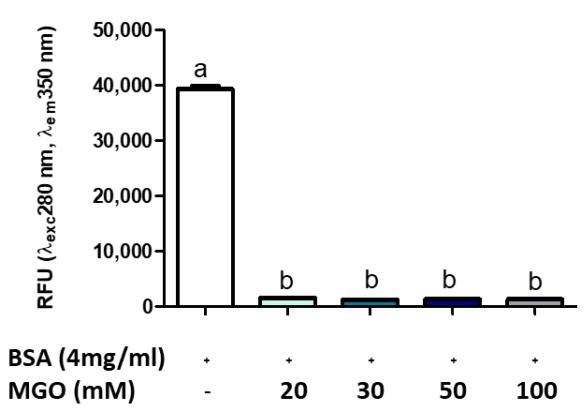

Figure 3. Fluorescence measurement of AGE after $168 \mathrm{~h}$ of incubation. All values are expressed as M $\pm \mathrm{SD}(n=3)$. Data represent relative fluorescence units (A) RFU $\lambda$ exc $365 \mathrm{~nm} / \lambda \mathrm{em} 440 \mathrm{~nm}$ and (B) RFU $\lambda$ exc $280 \mathrm{~nm} / \lambda$ em $350 \mathrm{~nm}$ of BSA non-glycated (BSA) and BSA glycated (BSA+MGO). Different letters represent significant differences among sample (ANOVA one-way and Tukey post-test).

A

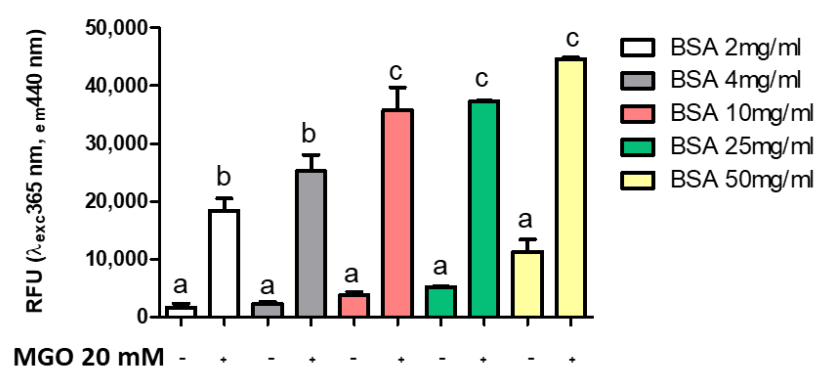

B

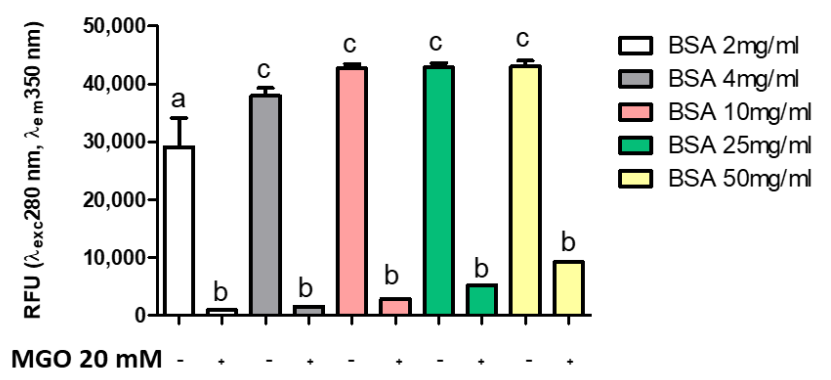

Figure 4. Fluorescence measurement of AGE after $168 \mathrm{~h}$ of incubation. All values are expressed as $\mathrm{M} \pm \mathrm{SD}(n=3)$. Data represent relative fluorescence units (A) RFU $\lambda$ exc $365 \mathrm{~nm} / \lambda \mathrm{em} 440 \mathrm{~nm}$ and (B) RFU $\lambda$ exc $280 \mathrm{~nm} / \lambda \mathrm{em} 350 \mathrm{~nm}$ of BSA non-glycated (BSA) and BSA glycated (BSA+MGO). Different letters represent significant differences among sample (ANOVA one-way and Tukey post-test).

The comparison of different fluorimetric analysis and timing suggests that, in our conditions (BSA $4 \mathrm{mg} / \mathrm{mL}$ as standard protein and MGO $20 \mathrm{mM}$ as glycosylated agent), the best time of incubation is seven days, since an incubation time over seven days did not determine a further increase in AGE formation (Figure S1). All fluorescent intensities were measured by a spectrophotometer (Infinite 200 Pro, Tecan, Männedorf, Switzerland).

\subsection{Inhibitory Effect of Hazelnut Skin Extract on AGEs}

During Maillard reaction, the carbonyl groups of a reducing sugar and free amino acid of proteins form Amadori products and various reactive dicarbonyl species such as 3-DG, GO, and MGO. MGO is the crucial intermediates to favor the formation of AGEs in vitro [50]. 
It is known that antioxidant activity of polyphenols relies on their capability to give electrons or hydrogen ions to free radical molecules as well as to free radicals' scavenging compounds [51]. Moreover, polyphenols can also react with lysine and arginine residues, thus inhibiting the bond between MGO or GO, two major precursors of AGEs, and free aminoacids [52]. The capability of reducing AGE formation has been reported for several phenolic molecules, which are not present or present only as minor components in hazelnut skin [27]. Ferulic acid inhibited the advanced phase of the glycation [22]; epicatechin, p-coumaric acid, and gallic acid decreased protein carbonyls and AGE formation [49]; protocatechuic acid, dihydroferulic acid, p-coumaric acid, p-hydroxybenzoic acid and salicylic acid showed strong inhibition of AGEs formation, with better results in an oleic acid-BSA system than in a glucose-BSA system [9]. Other compounds like (-)-epigallocatechin-3gallate (EGCG) [53] and proanthocyanidins [51] inhibited AGEs formation trapping MGO, with different flavonoids with vicinal dihydroxyl groups in the B-rings, such as miricitrin, rhamnetin, quercetin, and fisetin, inhibiting fluorescence AGE development [54]. Indeed, in the BSA-MGO system, quercetin traps MGO directly and then significantly inhibits the formation of AGEs [55].

There are two types of AGEs inhibitors: one is synthetic and the other is a natural compound. Both compounds have been assessed as inhibitors against the development of AGEs. Aminoguanidine (AG) or pimagedine is one well-known synthetic inhibitors of AGEs formation; it was first discovered in clinical trials. It traps reactive carbonyl precursors such as MGO, GO, and 3-DG to non-dangerous products, but this synthetic compound has several side effects [56]. The capability of hazelnut skin extract to inhibit AGE formation was also investigated and compared with that of gallic acid, the efficiency of which inhibiting the in vitro AGE formation is known [49], in addition to that of amminoguanidine.

The effect of hazelnut skin extract on the inhibition of AGE formation was dose dependent (Figure 5). Interestingly, hazelnut skin capacity to inhibit AGE formation is slightly and much higher than that of aminoguanidine and gallic acid, respectively, as it is evident by their IC50 (Table 4). The efficiency of a mix of bioactive molecules being higher than a single purified one has been reported for other molecules and biochemical/physiopathological processes [57].

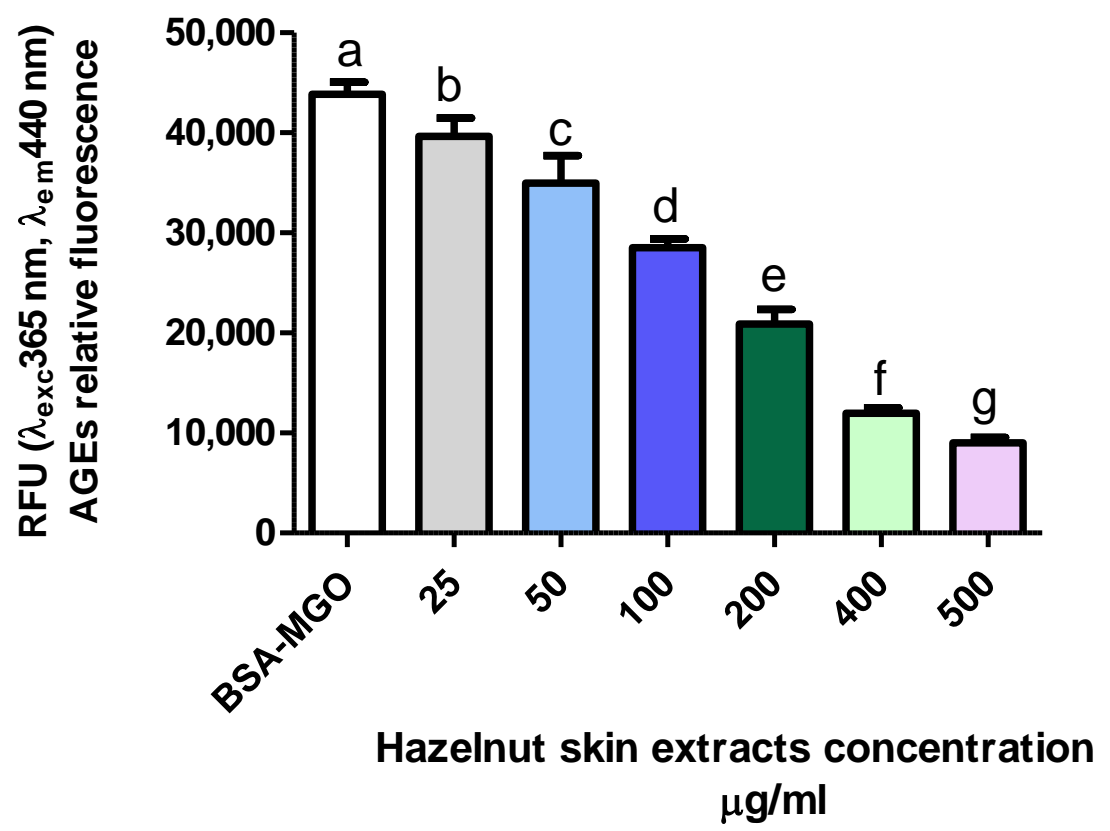

Figure 5. Inhibitory effect on AGE formation. Evaluation of total hazelnut extract $(\mathrm{Hz})$ dose-response effect on AGE formation in vitro. BSA-MGO represents the control group. Different letters represent significant differences among sample (ANOVA one-way and Tukey post-test). 
Table 4. IC50 value of synthetic or natural compounds: total hazelnut extract, gallic acid, and aminoguanidine. The concentration required for a 50\% inhibition of the intensity of fluorescence $(1=365 / 440 \mathrm{~nm})$ was calculated from the dose-inhibition curve, obtained by GraphPad analysis $(n=3)$.

\begin{tabular}{cc}
\hline Compounds & IC50 $(\mu \mathrm{g} / \mathrm{mL})$ \\
\hline Hz extract & 109.7 \\
gallic acid & 147.6 \\
AG & 117.8 \\
\hline
\end{tabular}

\section{Conclusions}

In this study, we have proven that the (poly)phenolic extract of hazelnut skins can reduce formation of AGEs in vitro. Our data set the basis for further investigation on the effects of hazelnut by-products in the prevention of potentially harmful glycation reactions, although other studies are needed to fully understand how these molecules interact with human physiological and pathological processes.

AGEs play key roles in cellular response to oxidative stress, through the regulation of different cell signaling pathways. Another class of closely related molecules, the advanced lipoxidation end-products (ALEs), plays alongside to AGEs in the establishment and progression of oxidative stress; elevated production of both ALEs and AGEs leads to protein cross-linking and aggregation, resulting in an alteration of cell signaling and functions, which leads to cell damage and death [58].

In this study, we have focused our attention on AGEs alone, although ALEs could represent a topic of interest for future investigation on the potentially healthy effects of bioactive compounds.

In this work, we have shown a potential role for an industrial by-product such as hazelnut skin for the extraction of bioactive compounds; this could represent an advantage for industries that have to deal with rather large amounts of these waste biomasses. The valorization of a waste with nutraceutical potential perfectly fits the circular bio-economy approach, in the ever-growing effort to reduce industrial waste and optimize every step of food production.

Supplementary Materials: The following are available online at https:/ /www.mdpi.com/2076-392 1/10/3/424/s1, Figure S1: Comparison of Fluorescence measurement of AGE at different time.

Author Contributions: Conceptualization, C.F., L.D. and L.D.G.; Data curation, L.S.; Formal analysis, L.S. and S.D.P.; Funding acquisition, L.D.G.; Investigation, L.S. and L.D.; Methodology, L.S., S.D.P., C.F., L.D. and L.D.G.; Project administration, L.D.G.; Resources, L.D.; Software, L.S., L.D. and L.D.G.; Supervision, C.F., L.D. and L.D.G.; Validation, C.F. and L.D.; Visualization, L.D. and L.D.G.; Writing—original draft, L.S. and L.D.; Writing—review and editing, L.S., L.D. and L.D.G. All authors have read and agreed to the published version of the manuscript.

Funding: This research was funded by "Soremartec Italia Srl" (Alba, Cuneo, Italy).

Institutional Review Board Statement: Not applicable.

Informed Consent Statement: Not applicable.

Data Availability Statement: The data presented in this study are available on request from the corresponding author.

Conflicts of Interest: The authors declare no conflict of interest.

\section{References}

1. Morand, C.; Tomás-Barberán, F.A. Contribution of plant food bioactives in promoting health effects of plant foods: Why look at interindividual variability? Eur. J. Nutr. 2019, 58, 13-19. [CrossRef]

2. Soare, A.; Khazrai, Y.M.; Fontana, L.; Del Toro, R.; Lazzaro, M.C.; Di Rosa, C.; Buldo, A.; Fioriti, E.; Maddaloni, E.; Angeletti, S.; et al. Treatment of reactive hypoglycemia with the macrobiotic Ma-PI 2 diet as assessed by continuous glucose monitoring: The MAHYP randomized crossover trial. Metabolism 2017, 69, 148-156. [CrossRef] 
3. Ran, X.L.; Zhang, M.; Wang, Y.; Adhikari, B. Novel technologies applied for recovery and value addition of high value compounds from plant byproducts: A review. Crit. Rev. Food. 2019, 59, 450-461. [CrossRef] [PubMed]

4. Shahidi, F.; Alasalvar, C.; Liyana-Pathirana, C.M. Antioxidant phytochemicals in hazelnut kernel (Corylus avellana L.) and hazelnut byproducts. J. Agric. Food Chem. 2007, 4, 1212-1220. [CrossRef] [PubMed]

5. Del Rio, D.; Calani, L.; Dall'Asta, M.; Brighenti, F. Polyphenolic composition of hazelnut skin. J. Agric. Food Chem. 2011, 59, 9935-9941. [CrossRef] [PubMed]

6. Yuan, B.; Lu, M.; Eskridge, K.M.; Isom, L.D.; Hanna, M.A. Extraction, identification, and quantification of antioxidant phenolics from hazelnut (Corylus avellana L.) shells. Food Chem. 2017, 244. [CrossRef] [PubMed]

7. Pelvan, E.; Olgun, E.Ö.; Karadağ, A.; Alasalvar, C. Phenolic profiles and antioxidant activity of Turkish Tombul hazelnut samples (natural, roasted, and roasted hazelnut skin). Food Chem. 2017, 244, 102-108. [CrossRef]

8. Masullo, M.; Cerulli, A.; Mari, A.; De Souza Santos, C.C.; Pizza, C.; Piacente, S. LC-MS profiling highlights hazelnut (Nocciola di Giffoni PGI) shells as a byproduct rich in antioxidant phenolics. Food Res. Int. 2017, 101, 180-187. [CrossRef] [PubMed]

9. Chen, H.; Virk, M.S.; Chen, F. Phenolic acids inhibit the formation of advanced glycation end products in food simulation systems depending on their reducing powers and structures. Int. J. Food Sci. Nutr. 2016, 67, 400-411. [CrossRef]

10. Khan, M.; Liu, H.; Wang, J.; Sun, B. Inhibitory effect of phenolic compounds and plant extracts on the formation of advance glycation end products: A comprehensive review. Food Res. Int. 2019, 130, 108933. [CrossRef]

11. Luevano-Contreras, C.; Chapman-Novakofski, K. Dietary advanced glycation end products and aging. Nutrient 2010, 2, 1247. [CrossRef]

12. Hemmler, D.; Roullier-Gall, C.; Marshall, J.V.; Rychlik, M.; Taylor, A.J.; Schmitt-Kopplin, P. Evolution of Complex Maillard Chemical Reactions, Resolved in Time. Sci. Rep. 2017, 7, 3-8. [CrossRef] [PubMed]

13. Vistoli, G.; De Maddis, D.; Cipak, A.; Zarkovic, N.; Carini, M.; Aldini, G. Advanced glycoxidation and lipoxidation end products (AGEs and ALEs): An overview of their mechanisms of formation. Free Radic. Res. 2013, 47, 3-27. [CrossRef] [PubMed]

14. Sell, D.R.; Strauch, C.M.; Shen, V.; Monnier, V.M. 2-Aminoadipic acid is a marker of protein carbonyl oxidation in the aging human skin: Effects of diabetes, renal failure and sepsis. Biochem. J. 2007, 404, 269-277. [CrossRef]

15. Schmitt, A.; Schmitt, J.; Münch, G.; Gasic-Milencovic, J. Characterization of advanced glycation end products for biochemical studies: Side chain modifications and fluorescence characteristics. Anal. Biochem. 2005, 338, 201-215. [CrossRef]

16. Bidasee, K.R.; Zhang, Y.; Shao, C.H.; Wang, M.; Patel, K.P.; Dincer, U.D.; Besch, H.R. Diabetes Increases Formation of Advanced Glycation End Products on Sarco(endo)plasmic Reticulum Ca2+-ATPase. Diabetes 2004, 5, 463-473. [CrossRef] [PubMed]

17. Hegab, Z.; Gibbons, S.; Neyses, L.; A Mamas, M. Role of advanced glycation end products in cardiovascular disease. World J. Cardiol. 2012, 4, 90. [CrossRef]

18. Chaudhuri, J.; Bains, Y.; Guha, S.; Kahn, A.; Hall, D.; Bose, N.; Gugliucci, A.; Kapahi, P. The Role of Advanced Glycation End Products in Aging and Metabolic Diseases: Bridging Association and Causality. Cell Metab. 2018, 28, 337-352. [CrossRef]

19. Thornalley, P.J. Use of aminoguanidine (Pimagedine) to prevent the formation of advanced glycation endproducts. Arch. Biochem. Biophys. 2003, 419, 31-40. [CrossRef]

20. Liu, L.; Hedegaard, R.V.; Skibsted, L.H. Effect of plant polyphenols on the formation of advanced glycation end products from ß-lactoglobulin. Food Sci. Biotechnol. 2017, 26, 389-391. [CrossRef]

21. Wu, J.V.; Hsieh, C.L.; Wang, H.Y.; Chen, H.Y. Inhibitory effects of guava (Psidium guajava L.) leaf extracts and its active compounds on the glycation process of protein. Food Chem. 2009, 113, 78-84. [CrossRef]

22. Silván, J.M.; Assar, S.H.; Srey, C.; Del Castillo, M.D.; Ames, J.M. Control of the Maillard reaction by ferulic acid. Food Chem. 2011, 128, 208-213. [CrossRef]

23. Umadevi, S.; Gopi, V.; Vellaichamy, E. Inhibitory effect of gallic acid on advanced glycation end products induced up-regulation of inflammatory cytokines and matrix proteins in H9C2 (2-1) cells. Cardiovasc. Toxicol. 2013, 13, 396-405. [CrossRef]

24. Mesías, M.; Navarro, M.; Gökmen, V.; Morales, F.J. Antiglycative effect of fruit and vegetable seed extracts: Inhibition of AGE formation and carbonyl-trapping abilities. J. Sci. Food Agric. 2013, 93, 2037-2044. [CrossRef]

25. Contini, M.; Baccelloni, S.; Massantini, R.; Anelli, G. Extraction of natural antioxidants from hazelnut (Corylus avellana L.) shell and skin wastes by long maceration at room temperature. Food Chem. 2008, 110, 659-669. [CrossRef]

26. Jakopic, J.; Petkovsek, M.M.; Likozar, A.; Solar, A.; Stampar, F.; Veberic, R. HPLC-MS identification of phenols in hazelnut (Corylus avellana L.) kernels. Food Chem. 2011, 124, 1100-1106. [CrossRef]

27. Fanali, C.; Tripodo, G.; Russo, M.; Della Posta, S.; Pasqualetti, V.; De Gara, L. Effect of solvent on the extraction of phenolic compounds and antioxidant capacity of hazelnut kernel. Electrophoresis 2018, 39, 1683-1691. [CrossRef]

28. Statista. Production of Tree Nuts Worldwide in 2019/2020, by Type (in 1000 Metric Tons). Available online: https:/ /www.sttista. com/statistics /1030790/tree-nut-global-production-by-type/ (accessed on 3 March 2021).

29. Martínez, M.; Moiraghi, L.; Agnese, M.; Guzman, C. Making and some properties of activated carbon produced from agricultural industrial residues from Argentina. J. Argent. Chem. Soc. 2003, 91, 104-108.

30. Barbu, M.C.; Sepperer, T.; Tudor, E.M.; Petutschnigg, A. Walnut and Hazelnut Shells: Untapped Industrial Resources and Their Suitability in Lignocellulosic Composites. Appl. Sci. 2020, 10, 6340. [CrossRef]

31. Alasalvar, A.; Hoffman, A.M.; Shahidi, F. Antioxidant Activities and Phytochemicals in Hazelnut (Corylus avellana L.) and Hazelnut By-Products. In Tree Nuts Composition, Phytochemicals, and Health Effects; Alasalvar, C., Shahidi, F., Eds.; CRC Press Taylor \& Francis Group: Boca Raton, FL, USA, 2008; pp. 215-235. 
32. Ainsworth, E.A.; Gillespie, K.M. Estimation of total phenolic content and other oxidation substrates in plant tissues using Folin-Ciocalteu reagent. Nat. Protoc. 2007, 875-877. [CrossRef]

33. Ellouze, I.; Abderrabba, M.; Sabaou, N.; Mathieu, F.; Lebrihi, A.; Bouajila, J. Season's Variation Impact on Citrus aurantium Leaves Essential Oil: Chemical Composition and Biological Activities. J. Food Sci. 2012, 77, 9. [CrossRef]

34. Huang, D.; Ou, B.; Hampsch-Woodill, M.; Flanagan, J.A.; Deemer, E.K. Development and validation of oxygen radical absorbance capacity assay for lipophilic antioxidants using randomly methylated $\beta$-cyclodextrin as the solubility enhancer. J. Agric. Food Chem. 2002, 50, 1815-1821. [CrossRef] [PubMed]

35. Starowicz, M.; Zieliński, H. Inhibition of advanced glycation end-product formation by high antioxidant-leveled spices commonly used in European cuisine. Antioxidants 2019, 8, 100. [CrossRef]

36. Özdemir, K.S.; Yilmaz, C.; Durmaz, G.; Gokmen, V. Hazelnut skin powder: A new brown colored functional ingredient. Food Res. Int. 2014, 65, 291-297. [CrossRef]

37. Slatnar, A.; Mikulic-Petkovsek, M.; Stampar, F.; Veberic, R.; Solar, A. HPLC-MSn identification and quantification of phenolic compounds in hazelnut kernels, oil and bagasse pellets. Food Res. Int. 2014, 64, 36. [CrossRef]

38. Monagas, M.; Garrido, I.; Lebron-Aguilar, R.; Gomez-Cordoves, M.C.; Rybarczyk, A.; Amarowicz, R.; Bartolome, B. Comparative flavan-3-ol profile and antioxidant capacity of roasted peanut, hazelnut, and almond skins. J. Agric. Food Chem. 2009, 57, 10590-10599. [CrossRef]

39. Piccinelli, A.L.; Pagano, I.; Esposito, T.; Mencherini, T.; Porta, A.; Petrone, A.M.; Gazzerro, P.; Picerno, P.; Sansone, P.; Rastrelli, L.; et al. HRMS Profile of a Hazelnut Skin Proanthocyanidin-rich Fraction with Antioxidant and Anti-Candida albicans Activities. J. Agric. Food Chem. 2016, 64, 585-595. [CrossRef] [PubMed]

40. Del Rio, D.; Rodriguez-Mateos, A.; Spencer, J.P.E.; Tognolini, M.; Borges, G.; Crozier, A. Dietary (poly)phenolics in human health: Structures, bioavailability, and evidence of protective effects against chronic diseases. Antioxid. Redox Signal 2013, 18, 1818-1892. [CrossRef]

41. Grosso, G.; Yang, J.; Marventano, S.; Micek, A.; Galvano, F.; Kales, S.N. Nut consumption on all-cause, cardiovascular, and cancer mortality risk: A systematic review and meta-analysis of epidemiologic studies. Am. J. Clin. Nutr. 2015, 101, 783-793. [CrossRef]

42. Mayhew, A.J.; De Souza, R.J.; Meyre, D.; Anand, S.S.; Mente, A. A systematic review and meta-analysis of nut consumption and incident risk of CVD and all-cause mortality. Br. J. Nutr. 2016, 115, 212-225. [CrossRef] [PubMed]

43. Contini, M.; Baccelloni, S.; Frangipane, M.T.; Merendino, M.; Massantini, R. Increasing espresso coffee brew antioxidant capacity using phenolic extract recovered from hazelnut skin waste. J. Funct. Foods. 2012, 4, 137-146. [CrossRef]

44. Anil, M. Using of hazelnut testa as a source of dietary fiber in breadmaking. J. Food Eng. 2007, 80, 61-67. [CrossRef]

45. Bertolino, M.; Belviso, S.; Dal Bello, B.; Ghirardello, D.; Giordano, M.; Rolle, L.; Gerbi, V.; Zeppa, G. Influence of the addition of different hazelnut skins on the physicochemical, antioxidant, polyphenol and sensory properties of yogurt. LWT Food Sci. Technol. 2015, 63, 1145-1154. [CrossRef]

46. Montella, R.; Coisson, J.D.; Travaglia, F.; Locatelli, M.; Mafalda, P.; Martelli, A.; Arlorio, M. Bioactive compounds from hazelnut skin (Corylus avellana L.): Effects on Lactobacillus plantarum P17630 and Lactobacillus crispatus P17631. J. Funct. Foods 2013, 5, 306-315. [CrossRef]

47. Caimari, A.; Puiggròs, F.; Suárez, M.; Crescenti, A.; Laos, S.; Ruiz, J.A.; Alonso, V.; Moragas, J.; Del Bas, J.M.; Arola, L. The intake of a hazelnut skin extract improves the plasma lipid profile and reduces the lithocholic/deoxycholic bile acid faecal ratio, a risk factor for colon cancer, in hamsters fed a high-fat diet. Food Chem. 2015, 167, 138-144. [CrossRef] [PubMed]

48. Ivanovic, S.; Avramovic, N.; Dojcinovic, B.; Trifunovic, S.; Novakovic, M.; Teševic, V.; Mandic, B. Chemical composition, total phenols and flavonoids contents and antioxidant activity as nutritive potential of roasted hazelnut skins (Corylus avellana L.). Foods 2020, 9, 430. [CrossRef] [PubMed]

49. Ardestani, A.; Yazdanparast, R. Cyperus rotundus suppresses AGE formation and protein oxidation in a model of fructosemediated protein glycoxidation. Int. J. Biol. Macromol. 2007, 41, 572-578. [CrossRef]

50. Ou, J.; Huang, J.; Wang, M.; Ou, S. Effect of rosmarinic acid and carnosic acid on AGEs formation in vitro. Food Chem. 2017, 221, 1057-1061. [CrossRef]

51. Peng, X.; Ma, J.; Chen, F.; Wang, M. Naturally occurring inhibitors against the formation of advanced glycation end-product. Food Funct. 2001, 2, 289-301. [CrossRef] [PubMed]

52. Nagaraj, R.H.; Sarkar, P.; Mally, A.; Biemel, K.M.; Lederer, O.; Padayatti, P.S. Effect of pyridoxamine on chemical modification of proteins by carbonyls in diabetic rats: Characterization of a major product from the reaction of pyridoxamine and methylglyoxal. Arch. Biochem. Biophys. 2002, 402, 110-119. [CrossRef]

53. Yoon, S.P.; Maeng, Y.H.; Hong, R.; Byung, R.L.; Lee, B.R.; Kime, G.K.; Kim, C.G.; Chung, J.H.; Shin, B.C. Protective effects of epigallocatechin gallate (EGCG) on streptozotocin-induced diabetic nephropathy in mice. Acta Histochem. 2014, 116, 1210-1215. [CrossRef] [PubMed]

54. Cervantes-Laurean, D.; Schramm, D.D.; Jacobson, E.L.; Halaweish, I.; Bruckner, G.G.; Boissonneault, G.A. Inhibition of advanced glycation end product formation on collagen by rutin and its metabolites. J. Nutr. Biochem. 2006, 17, 531-540. [CrossRef] [PubMed]

55. Li, X.; Zheng, T.; Sang, S.; Lv, L. Quercetin inhibits advanced glycation end product formation by trapping methylglyoxal and glyoxal. J. Agric. Food Chem. 2014, 62, 152-12158. [CrossRef]

56. Jud, P.; Sourij, H. Therapeutic options to reduce advanced glycation end products in patients with diabetes mellitus: A review. Diabetes Res. Clin. Pract. 2019, 148, 54-63. [CrossRef] [PubMed] 
57. D’Introno, A.; Paradiso, A.; Scoditti, E.; D'Amico, L.; De Paolis, A.; Carluccio, M.A.; Nicoletti, I.; De Gara, L.; Santino, A.; Giovinazzo, G. Antioxidant and anti-inflammatory properties of tomato fruits synthesizing different amounts of stilbenes. Plant Biotechnol. J. 2009, 7, 422-429. [CrossRef]

58. Moldogazieva, N.T.; Mokhosoev, I.M.; Mel'nikova, T.I.; Porozov, Y.B.; Terentiev, A.A. Oxidative Stress and Advanced Lipoxidation and Glycation End Products (ALEs and AGEs) in Aging and Age-Related Diseases. Oxidative Med. Cell. Longev. 2019, $2019,1-14$. [CrossRef] 